\title{
THE DISTANCE FUNCTION FROM THE BOUNDARY OF A DOMAIN WITH CORNERS
}

\author{
MOHAMMAD SAFDARI
}

\begin{abstract}
We study the regularity of the distance function to the boundary of a domain in $\mathbb{R}^{2}$, with respect to some asymmetric norms. We allow the boundary of the domain to have corners. We obtain an explicit formula for the second derivative of these distance functions. Furthermore, we study a generalized notion of the ridge of a domain, which is the set of singularities of a distance function to the boundary of the domain. We completely characterize the ridge by using a generalized notion of curvature.
\end{abstract}

\section{INTRODUCTION}

The distance function from the boundary of a domain appears in different parts of mathematical analysis, especially in the study of partial differential equations. Crasta and Malusa [4] studied the distance function, and used it to analyze PDEs of Monge-Kantorovich type arising in optimal transport theory. Li and Nirenberg [6] studied the distance function in the framework of HamiltonJacobi equations and Finsler geometry. Itoh and Tanaka [5], and Mantegazza and Mennucci [7] studied the distance function in Riemannian manifolds; and Chruściel et al. [2] considered the case of Lorentzian space-times. On the other hand, Clarke et al. [3] and Poliquin et al. [8] studied the distance function in the context of nonsmooth analysis in Hilbert spaces.

In 9 12], we examined the distance function, and used it to study variational problems with gradient constraint, and their corresponding free boundary. Let us introduce this problem in more detail, and see how we utilized the distance function in its study. Let $K$ be a compact convex subset of $\mathbb{R}^{n}$ whose interior contains the origin. We recall from convex analysis (see [13]) that the gauge function of $K$ is the convex function

$$
\gamma_{K}(x):=\inf \{\lambda>0: x \in \lambda K\}
$$

The gauge function $\gamma_{K}$ is subadditive and positively 1-homogenous, so it looks like a norm on $\mathbb{R}^{n}$, except that $\gamma_{K}(-x)$ is not necessarily the same as $\gamma_{K}(x)$. Another notion is that of the polar of $K$

$$
K^{\circ}:=\{x:\langle x, y\rangle \leq 1 \text { for all } y \in K\}
$$

where $\langle$,$\rangle is the standard inner product on \mathbb{R}^{n}$. $K^{\circ}$, too, is a compact convex set containing the origin as an interior point.

School of Mathematics, Institute for Research in Fundamental Sciences (IPM), P.O. Box: 19395-5746, Tehran, Iran Email address: safdari@sharif.ir. 
Let $U \subset \mathbb{R}^{n}$ be a bounded open set. Let $u$ be the minimizer of

$$
J[v]:=\int_{U} F(D v)+g(v) d x
$$

over

$$
W_{K^{\circ}}:=\left\{v \in H_{0}^{1}(U): D v \in K^{\circ} \text { a.e. }\right\} \text {. }
$$

Note that $D v \in K^{\circ}$ is equivalent to $\gamma_{K^{\circ}}(D v) \leq 1$. Thus $\gamma_{K^{\circ}}$ defines the gradient constraint. It can be shown that $u$ is also the unique minimizer of $J$ over

$$
W_{d_{K}}:=\left\{v \in H_{0}^{1}(U):-\bar{d}_{K} \leq v \leq d_{K} \text { a.e. }\right\},
$$

where

$$
\begin{aligned}
& d_{K}(x)=d_{K}(x, \partial U):=\min _{y \in \partial U} \gamma_{K}(x-y), \\
& \bar{d}_{K}(x)=\bar{d}_{K}(x, \partial U):=\min _{y \in \partial U} \gamma_{K}(y-x) .
\end{aligned}
$$

Note that $\bar{d}_{K}=d_{-K}$, since $\gamma_{-K}(\cdot)=\gamma_{K}(-\cdot)$. Thus the distance function from $\partial U$ with respect to some gauge function naturally appears in the study of variational problems with gradient constraint.

In [9, 10] we investigated the above problem in two dimensions, when $K$ is given by the $p$-norm, i.e. when $K$ is equal to

$$
\left\{\left(x_{1}, x_{2}\right):\left(\left|x_{1}\right|^{p}+\left|x_{2}\right|^{p}\right)^{\frac{1}{p}} \leq 1\right\} .
$$

In this regard, we had to understand the behavior of $d_{K}$. In [12] we generalized the above work to arbitrary dimensions, for arbitrary gradient constraints. Here, we approximated an arbitrary convex set $K$ with convex sets $K_{i}$ whose boundaries are $C^{2}$, and have positive principal curvatures. This analysis relied heavily on the properties of $d_{K}$ and $d_{K_{i}}$. Especially, we were able to find an explicit formula for $D^{2} d_{K_{i}}$, which was very crucial in our analysis. To the best of author's knowledge, formulas of this kind have not appeared in the literature before, except for the simple case of the Euclidean distance to the boundary.

In this work, we examine the regularity of $d_{K}$ in two dimensions. We obtain formula (3.2) for $D^{2} d_{K}$, when $\partial K$ is $C^{2}$. In contrast to [12], here we allow the curvature of $\partial K$ to vanish at finitely many points. Hence this work includes the case of $p$-norms as a special case. Also, here we allow $\partial U$ to have corners; which makes the behavior of $d_{K}$ more complicated. In addition, we will completely characterize the set of singularities of $d_{K}$, which we call the $d_{K}$-ridge.

The paper is organized as follows. In Section 2 we introduce our notation, and review some preliminary facts about $d_{K}$ and $\gamma_{K}$. In Section 3 we will study the regularity of $d_{K}$. Theorems 1. 2 are the main results of this section; and provide detailed information about $d_{K}$. Here, we also introduce a generalized notion of curvature, which helps us to understand the behavior of $d_{K}$. Finally, in Section 4 we will characterize the set of singularities of $d_{K}$.

\section{Notation And Preliminaries}

Let us fix some notation first. We denote by $C^{\omega}$ the space of analytic functions (or submanifolds); so in the following when we talk about $C^{k, \alpha}$ regularity with $k$ greater than some fixed integer, we 
are also including $C^{\infty}$ and $C^{\omega}$. We will use the abbreviations

$$
\gamma:=\gamma_{K}, \quad \gamma^{\circ}:=\gamma_{K^{\circ}} .
$$

We also denote the closed line segment between two points $x, y$ by $[x, y]$, the open line segment by ]$x, y[$, and the half-closed line segments by $] x, y],\left[x, y\left[\right.\right.$. When $d_{K}(x)=\gamma(x-y)$ for some $y \in \partial U$, we call $y$ a $\boldsymbol{d}_{\boldsymbol{K}}$-closest point to $x$ on $\partial U$. Note that when $y$ is a $d_{K}$-closest point on $\partial U$ to $x \in U$, the segment $\left[x, y\left[\right.\right.$ is in $U$. We also use $B_{r}(x)$ to denote the open ball of radius $r$ centered at $x$.

For $X \subset \mathbb{R}^{n}, v \in \mathbb{R}^{n}$, and $r \in \mathbb{R}$ we use the conventions

$$
\begin{aligned}
& r X:=\{r x \mid x \in X\}, \\
& v+X:=\{v+x \mid x \in X\} .
\end{aligned}
$$

When $n=2$, we use the notation $v^{\perp}:=\left(-v_{2}, v_{1}\right)$ for the $90^{\circ}$ counterclockwise rotation of a vector $v=\left(v_{1}, v_{2}\right)$. We also set $D^{\perp} f:=(D f)^{\perp}$ for a function $f$ of two variables.

2.1. The ridge. First, we generalize the notion of ridge introduced by Ting [14], and Caffarelli and Friedman [1].

Definition 1. The $\boldsymbol{d}_{\boldsymbol{K}}$-ridge of $U$ is the set of all points $x \in U$ where $d_{K}(x)=d_{K}(x, \partial U)$ is not $C^{1,1}$ in any neighborhood of $x$. We denote it by

$$
R_{K}
$$

Recall that $K$ is a compact convex subset of $\mathbb{R}^{n}$ with 0 in its interior, and its gauge function $\gamma$ satisfies

$$
\begin{aligned}
& \gamma(r x)=r \gamma(x), \\
& \gamma(x+y) \leq \gamma(x)+\gamma(y),
\end{aligned}
$$

for all $x, y \in \mathbb{R}^{n}$ and $r \geq 0$. Note that as $K$ is closed, $K=\{\gamma \leq 1\}$; and as it has nonempty interior, $\partial K=\{\gamma=1\}$. Thus, $\gamma(x-y) \leq r$ is equivalent to $y \in x-r K$. Also, note that as $B_{c}(0) \subseteq K \subseteq B_{C}(0)$ for some $C \geq c>0$, we have

$$
\frac{1}{C}|x| \leq \gamma(x) \leq \frac{1}{c}|x|,
$$

for all $x \in \mathbb{R}^{n}$. Moreover, from the definition of $d_{K}$ we easily obtain

$$
-\gamma(x-y) \leq d_{K}(y)-d_{K}(x) \leq \gamma(y-x) .
$$

Thus in particular, $d_{K}$ is Lipschitz continuous.

It is well known that for all $x, y \in \mathbb{R}^{n}$, we have

$$
\langle x, y\rangle \leq \gamma(x) \gamma^{\circ}(y) .
$$

In fact, more is true and we have

$$
\gamma^{\circ}(y)=\max _{x \neq 0} \frac{\langle x, y\rangle}{\gamma(x)} .
$$

For a proof of this, see page 54 of [13]. 
It is easy to see that the the strict convexity of $K$ (which means that $\partial K$ does not contain any line segment) is equivalent to the strict convexity of $\gamma$. By homogeneity of $\gamma$, the latter is equivalent to

$$
\gamma(x+y)<\gamma(x)+\gamma(y)
$$

when $x \neq c y$ and $y \neq c x$ for any $c \geq 0$.

The following three lemmas do not require any assumption about $\partial U$.

Lemma 1. Suppose $y$ is one of the $d_{K}$-closest points on $\partial U$ to $x \in U$. Then $y$ is a $d_{K}$-closest point on $\partial U$ to every point of $] x, y\left[\right.$. If in addition $\gamma$ is strictly convex, then $y$ is the unique $d_{K}$-closest point on $\partial U$ to points of $] x, y[$.

Proof. Let $z \in] x, y[$, and suppose to the contrary that there is $w \in \partial U-\{y\}$ such that

$$
\gamma(z-w)<\gamma(z-y) \text {. }
$$

Then we have

$$
\gamma(x-w) \leq \gamma(x-z)+\gamma(z-w)<\gamma(x-z)+\gamma(z-y)=\gamma(x-y) .
$$

Which is a contradiction.

Now suppose $\gamma$ is strictly convex, and

$$
\gamma(z-w) \leq \gamma(z-y)
$$

If $w$ belongs to the line containing $x, z, y$, then considering the order of these four points on that line, we can easily arrive at a contradiction. Hence, $x, z, w$ are not collinear, and by strict convexity of $\gamma$ we get

$$
\gamma(x-w)<\gamma(x-z)+\gamma(z-w) \leq \gamma(x-z)+\gamma(z-y)=\gamma(x-y) .
$$

Which is a contradiction too.

Lemma 2. Suppose $\gamma$ is strictly convex. If $d_{K}(x)=\gamma(x-y)=\gamma(x-z)$ for two different points $y, z$ on $\partial U$, then $d_{K}$ is not differentiable at $x$.

Proof. The points in the segment $[x, y]$ have $y$ as $d_{K}$-closest point on $\partial U$. Hence for $0 \leq t \leq \gamma(x-y)$ we have

$$
\begin{aligned}
d_{K}\left(x-\frac{t}{\gamma(x-y)}(x-y)\right) & =\gamma\left(x-\frac{t}{\gamma(x-y)}(x-y)-y\right) \\
& =\left(1-\frac{t}{\gamma(x-y)}\right) \gamma(x-y)=\gamma(x-y)-t .
\end{aligned}
$$

Now suppose to the contrary that $d_{K}$ is differentiable at $x$. Then by differentiating the above equality with respect to $t$ (and the similar formula for $z$ ), we get

$$
\left\langle D d_{K}(x), \frac{x-y}{\gamma(x-y)}\right\rangle=1=\left\langle D d_{K}(x), \frac{x-z}{\gamma(x-z)}\right\rangle .
$$

On the other hand, it is easy to show that $\gamma^{\circ}\left(D d_{K}(x)\right) \leq 1$. To do this, just note that

$$
d_{K}(x+t v)-d_{K}(x) \leq \gamma(x+t v-x)=t \gamma(v) .
$$

Taking the limit as $t \rightarrow 0^{+}$, we get $\left\langle D d_{K}(x), v\right\rangle \leq \gamma(v)$. We get the desired by (2.3). 
Now note that there is at most one vector $v$ with $\gamma(v)=1$ such that

$$
\left\langle D d_{K}(x), v\right\rangle=1 \text {. }
$$

Since, otherwise for two such vectors $v, w$, we would have $\left\langle D d_{K}(x),\left(\frac{v+w}{2}\right)\right\rangle=1$. However, by strict convexity of $\gamma$, and inequality (2.2), we get

$$
\begin{aligned}
\left\langle D d_{K}(x), \frac{v+w}{2}\right\rangle & \leq \gamma^{\circ}\left(D d_{K}(x)\right) \gamma\left(\frac{v+w}{2}\right) \\
& <\gamma^{\circ}\left(D d_{K}(x)\right) \frac{\gamma(v)+\gamma(w)}{2}=1 .
\end{aligned}
$$

Which is a contradiction. Therefore $d_{K}$ can not be differentiable at $x$.

Definition 2. For a strictly convex $K$, the subset of the $d_{K}$-ridge consisting of the points with more than one $d_{K}$-closest point on $\partial U$, is denoted by

$$
R_{K, 0} \cdot
$$

Lemma 3. Suppose $x_{i} \in \bar{U}$ converge to $x \in \bar{U}$, and $y \in \partial U$ is the unique $d_{K}$-closest point to $x$. If $y_{i} \in \partial U$ is a (not necessarily unique) $d_{K}$-closest point to $x_{i}$, then $y_{i}$ converges to $y$.

If $x$ has more than one $d_{K}$-closest point on $\partial U$, and $y_{i}$ converges to $\tilde{y} \in \partial U$, then $\tilde{y}$ is one of the $d_{K}$-closest points on $\partial U$ to $x$.

Proof. Suppose that the claim of the first part does not hold. Then a subsequence of $y_{i}$, which we still denote it by $y_{i}$, will remain outside an open ball $B$ around $y$. Now consider the set $L:=x-d_{K}(x) K$ that touches $\partial U$ only at $y$. Since $L$ is a compact set inside the open set $U \cup B$, a set of the form $x-\left(d_{K}(x)+\varepsilon\right) K$ is still inside $U \cup B$. Now, let $\epsilon<\frac{\varepsilon}{2}, d_{K}(x)$. As $x_{i}$ 's approach $x$, they will be inside $x-\epsilon K$ eventually. Therefore

$$
d_{K}(x)+\varepsilon \leq \gamma\left(x-y_{i}\right) \leq \gamma\left(x-x_{i}\right)+\gamma\left(x_{i}-y_{i}\right)<\epsilon+\gamma\left(x_{i}-y_{i}\right) .
$$

Hence

$$
d_{K}(x)+\frac{\varepsilon}{2}<d_{K}(x)+\varepsilon-\epsilon<d_{K}\left(x_{i}\right) .
$$

But this contradicts the continuity of $d_{K}$.

Now let us consider the second statement. If the claim fails, then $\tilde{y}$ is outside the compact set $L$. We can enlarge $L$ to $x-\left(d_{K}(x)+\varepsilon\right) K$ so that $\tilde{y}$ is still outside the enlarged set. Now, let $\epsilon<\frac{\varepsilon}{3}, d_{K}(x)$. As $x_{i} \rightarrow x$ and $y_{i} \rightarrow \tilde{y}$, they will be respectively inside $x-\epsilon K$ and $y-\epsilon K$ eventually. Thus

$$
d_{K}(x)+\varepsilon \leq \gamma(x-\tilde{y}) \leq \gamma\left(x-x_{i}\right)+\gamma\left(x_{i}-y_{i}\right)+\gamma\left(y_{i}-\tilde{y}\right)<2 \epsilon+\gamma\left(x_{i}-y_{i}\right) .
$$

Which gives a contradiction as above.

2.2. Regularity of the gauge function. Remember that $K$ is a compact convex subset of $\mathbb{R}^{n}$ whose interior contains the origin. Suppose that $\partial K$ is $C^{k, \alpha}(k \geq 2,0 \leq \alpha \leq 1)$. Let us show that as a result, $\gamma$ is $C^{k, \alpha}$ on $\mathbb{R}^{n}-\{0\}$. Let $r=\rho(\theta)$ for $\theta \in \mathbb{S}^{n-1}$, be the equation of $\partial K$ in polar coordinates. Then $\rho$ is positive and $C^{k, \alpha}$. To see this note that locally, $\partial K$ is given by a $C^{k, \alpha}$ 
equation $f(x)=0$. On the other hand we have $x=r X(\theta)$, for some smooth function $X$. Hence we have $f(r X(\theta))=0$; and the derivative of this expression with respect to $r$ is

$$
\langle X(\theta), D f(r X(\theta))\rangle=\frac{1}{r}\langle x, D f(x)\rangle .
$$

But this is nonzero since $D f$ is orthogonal to $\partial K$, and $x$ cannot be tangent to $\partial K$ (otherwise 0 cannot be in the interior of $K$, as $K$ lies on one side of its supporting hyperplane at $x$ ). Thus we get the desired by the Implicit Function Theorem. Now, it is straightforward to check that for a nonzero point in $\mathbb{R}^{n}$ with polar coordinates $(s, \phi)$ we have

$$
\gamma((s, \phi))=\frac{s}{\rho(\phi)}
$$

This formula easily gives the smoothness of $\gamma$.

Remark 1 . The above argument works when $k=1$ too, but we need the extra regularity for what follows. Also note that as $\partial K=\{\gamma=1\}$ and $D \gamma \neq 0$ by (2.4), $\partial K$ is as smooth as $\gamma$.

Now, suppose in addition that $K$ is strictly convex. Then $\gamma$ is strictly convex too. By Remark 1.7.14 and Theorem 2.2.4 of [13], $K^{\circ}$ is also strictly convex and its boundary is $C^{1}$. Therefore $\gamma^{\circ}$ is strictly convex, and it is $C^{1}$ on $\mathbb{R}^{n}-\{0\}$. Thence by Corollary 1.7.3 of [13], for $x \neq 0$ we have

$$
D \gamma(x) \in \partial K^{\circ}, \quad D \gamma^{\circ}(x) \in \partial K
$$

In particular $D \gamma, D \gamma^{\circ}$ are nonzero on $\mathbb{R}^{n}-\{0\}$.

We also suppose that the smallest principal curvature of $\partial K$ is positive everywhere except possibly at a finite number of points where it vanishes. Let $\left\{\mu_{1}, \ldots, \mu_{m}\right\}$ be the outward unit normal to $\partial K$ at these points.

We can show that $\gamma^{\circ}$ is $C^{k, \alpha}$ on $\mathbb{R}^{n}-\left\{t \mu_{i}: t \geq 0, i=1, \ldots, m\right\}$. To see this, let $n_{K}: \partial K \rightarrow \mathbb{S}^{n-1}$ be the Gauss map, i.e. $n_{K}(y)$ is the outward unit normal to $\partial K$ at $y$. Then $n_{K}$ is $C^{k-1, \alpha}$ and its derivative is an isomorphism at the points with positive principal curvatures. Hence $n_{K}$ is locally invertible with a $C^{k-1, \alpha}$ inverse $n_{K}^{-1}$, around any point of $\mathbb{S}^{n-1}-\left\{\mu_{1}, \ldots, \mu_{m}\right\}$. Now note that as it is well known, $\gamma^{\circ}$ equals the support function of $K$, i.e.

$$
\gamma^{\circ}(x)=\sup \{\langle x, y\rangle: y \in K\} .
$$

Thus as shown on page 115 of [13], for $x \neq 0$ we have

$$
D \gamma^{\circ}(x)=n_{K}^{-1}\left(\frac{x}{|x|}\right)
$$

Which gives the desired result. As a consequence, since $\partial K^{\circ}=\left\{\gamma^{\circ}=1\right\}$ and $D \gamma^{\circ} \neq 0$ by (2.4), $\partial K^{\circ}$ is $C^{k, \alpha}$ except possibly at finitely many points which are positive multiples of $\mu_{i}$ 's.

Let us recall a few more properties of $\gamma, \gamma^{\circ}$. Since they are positively 1-homogenous, $D \gamma, D \gamma^{\circ}$ are positively 0-homogenous, and $D^{2} \gamma, D^{2} \gamma^{\circ}$ (the latter when exists) are positively (-1)-homogenous, i.e.

$$
\begin{array}{ccc}
\gamma(t x)=t \gamma(x), & D \gamma(t x)=D \gamma(x), & D^{2} \gamma(t x)=\frac{1}{t} D^{2} \gamma(x), \\
\gamma^{\circ}(t x)=t \gamma^{\circ}(x), & D \gamma^{\circ}(t x)=D \gamma^{\circ}(x), & D^{2} \gamma^{\circ}(t x)=\frac{1}{t} D^{2} \gamma^{\circ}(x),
\end{array}
$$


for $x \neq 0$ and $t>0$. As a result, using Euler's theorem on homogenous functions we get

$$
\begin{array}{cl}
\langle D \gamma(x), x\rangle=\gamma(x), & D^{2} \gamma(x) x=0, \\
\left\langle D \gamma^{\circ}(x), x\right\rangle=\gamma^{\circ}(x), & D^{2} \gamma^{\circ}(x) x=0,
\end{array}
$$

for $x \neq 0$. Note that in both (2.5), (2.6) we need to assume $x \neq t \mu_{i}$ for any $t>0$, when dealing with $D^{2} \gamma^{\circ}$. We also recall the following fact from [4], that for $x \neq 0$

$$
D \gamma^{\circ}(D \gamma(x))=\frac{x}{\gamma(x)}, \quad D \gamma\left(D \gamma^{\circ}(x)\right)=\frac{x}{\gamma^{\circ}(x)} \text {. }
$$

Remark 2. Let us assume for simplicity that $n=2$. As a consequence of (2.6), we see that if $x \neq t \mu_{i}$ for any $t>0$, then it is an eigenvector of $D^{2} \gamma^{\circ}(x)$ with eigenvalue 0 . Since $D^{2} \gamma^{\circ}(x)$ is a symmetric matrix, its other eigenvector can be taken to be $x^{\perp}$. By Corollary 2.5.2 of [13] and (-1)-homogeneity of $D^{2} \gamma^{\circ}$, the other eigenvalue of $D^{2} \gamma^{\circ}(x)$ is

$$
\frac{1}{|x|} r_{K}\left(n_{K}^{-1}\left(\frac{x}{|x|}\right)\right)
$$

Here $r_{K}$ is the radius of curvature of $\partial K$, i.e. the reciprocal of its curvature; and $n_{K}^{-1}$ is the inverse of the Gauss map of $\partial K$. Hence, the eigenvalues of $D^{2} \gamma^{\circ}(x)$ are 0 and a positive number.

\section{Regularity of the Distance FunCtion}

In this section, we are going to study the singularities of the function $d_{K}$. It is obvious that $d_{K}$ is a Lipschitz function. We want to characterize the set over which it is more regular. In order to do that, we need to impose some restrictions on $\partial K, \partial U$.

For the rest of this paper we assume that $n=2$. Let $U \subset \mathbb{R}^{2}$ be a bounded open set, whose boundary is the union of simple closed Jordan curves consisting of $\operatorname{arcs} S_{1}, \ldots, S_{N}$ which are $C^{k, \alpha}$ $(k \geq 2,0 \leq \alpha \leq 1)$ up to their endpoints, satisfying Assumption 1 below. Thus, topologically, $U$ is homeomorphic to the interior of a disk from which, possibly, several disks are removed. If $S_{i} \cap S_{j}$ is nonempty, in which case it consists of a single point, we call that point a corner or a vertex of $\partial U$. A nonreentrant corner of $\partial U$ is a corner whose opening angle is less than $\pi$. And, a reentrant corner is a corner with opening angle greater than or equal to $\pi$. If the angle of a reentrant corner is strictly greater than $\pi$ we call it a strict reentrant corner. We assume that the opening angles of the vertices of $\partial U$ are strictly between 0 and $2 \pi$, i.e. there are no cusps. As a result, $\partial U$ is locally the graph of a Lipschitz function.

Remark 3. We can allow cusps with angle 0 in Theorem 1, and arbitrary cusps in Theorems 2 , 4 and 5. But we need the Lipschitz regularity of $\partial U$ when we deal with the variational problem.

Assumption 1. Let $y \in S_{i}$ be an interior point of $S_{i}$, or a reentrant corner. We assume that if the inward unit normal to $S_{i}$ at $y$ belongs to $\left\{\mu_{1}, \ldots, \mu_{m}\right\}$, then either the curvature of $S_{i}$ at $y$ is positive, or $S_{i}$ is a line segment.

Note that there are at most finitely many points on each $S_{i}$ at which the inward unit normal belongs to $\left\{\mu_{1}, \ldots, \mu_{m}\right\}$, and the curvature of $S_{i}$ at them is positive. The reason is that these points are isolated; because the derivative of the inward normal at them is nonzero, due to the positivity of the curvature (see (3.1)). 
First we assume that all the corners of $\partial U$ are nonreentrant. We will consider domains with reentrant corners later.

Next, we introduce a new notion of curvature for curves in the plane. It will be used to study the regularity of $d_{K}$.

Definition 3. The $\boldsymbol{K}$-curvature of a $C^{2}$ curve $t \mapsto(x(t), y(t))$ in the plane is

$$
\kappa_{K}:=\frac{1}{|\nu|^{2}}\left\langle D^{2} \gamma^{\circ}(\nu) \nu^{\prime}, \nu^{\perp}\right\rangle
$$

Here, $\nu:=\left(-y^{\prime}, x^{\prime}\right)$ is normal to the curve, and $D^{2} \gamma^{\circ}(\nu) \nu^{\prime}$ is the action of the matrix $D^{2} \gamma^{\circ}(\nu)$ on the vector $\nu^{\prime}$. We assume that $\nu$ is nonzero and is not a positive multiple of any of $\mu_{i}{ }^{\prime}$ s. When the curve is a line segment and $\nu \equiv c \mu_{i}$ for some $c>0$, we define $\kappa_{K} \equiv 0$.

It is easy to see that $\kappa_{K}$ does not change under reparametrizations of the curve, hence it is an intrinsic quantity. Also note that $\left\langle\nu^{\prime}, \nu^{\perp}\right\rangle=\kappa|\nu|^{3}$, where $\kappa$ is the ordinary curvature.

Lemma 4. We have

$$
\begin{aligned}
& D^{2} \gamma^{\circ}(\nu) \nu^{\prime}=\kappa_{K} \nu^{\perp}, \\
& \kappa_{K}=\frac{1}{\gamma^{\circ}(\nu)}\left\langle D^{2} \gamma^{\circ}(\nu) \nu^{\prime}, D^{\perp} \gamma^{\circ}(\nu)\right\rangle .
\end{aligned}
$$

Proof. Since we have $D^{2} \gamma^{\circ}(\nu) \nu=0$, and $D^{2} \gamma^{\circ}$ is a symmetric matrix, we get

$$
\left\langle\left(D^{2} \gamma^{\circ} \nu^{\prime}\right)^{\perp}, \nu^{\perp}\right\rangle=\left\langle D^{2} \gamma^{\circ} \nu^{\prime}, \nu\right\rangle=\left\langle\nu^{\prime}, D^{2} \gamma^{\circ} \nu\right\rangle=0 .
$$

Thus $D^{2} \gamma^{\circ}(\nu) \nu^{\prime}$ is parallel to $\nu^{\perp}$, and from the definition of $K$-curvature we get $D^{2} \gamma^{\circ}(\nu) \nu^{\prime}=\kappa_{K} \nu^{\perp}$.

Then by (2.6) we get

$$
\left\langle D^{2} \gamma^{\circ} \nu^{\prime}, D^{\perp} \gamma^{\circ}\right\rangle=\left\langle\kappa_{K} \nu^{\perp}, D^{\perp} \gamma^{\circ}\right\rangle=\kappa_{K}\left\langle\nu, D \gamma^{\circ}\right\rangle=\kappa_{K} \gamma^{\circ}(\nu)
$$

Lemma 5. $\kappa_{K}$ has the same sign as the ordinary curvature $\kappa$. In particular, $\kappa_{K}=0$ if and only if $\kappa=0$.

Proof. We can write $\nu^{\prime}$ as a linear combination of $\nu, \nu^{\perp}$

$$
\nu^{\prime}=a \nu+b \nu^{\perp} .
$$

Since by (2.8) we know that $D^{2} \gamma^{\circ}(\nu) \nu^{\perp}=\lambda \nu^{\perp}$ for some $\lambda>0$, using (2.6), (3.1) we get

$$
\kappa_{K} \nu^{\perp}=D^{2} \gamma^{\circ}(\nu) \nu^{\prime}=\lambda b \nu^{\perp} .
$$

On the other hand $\kappa=\frac{\left\langle\nu^{\prime}, \nu^{\perp}\right\rangle}{|\nu|^{3}}=\frac{b}{|\nu|}$. Therefore

$$
\kappa_{K}=|\nu| \lambda \kappa
$$

Remark 4. By (2.8), the interpretation of the above formula is that the $K$-curvature at a point with normal $\nu$, is the ordinary curvature at that point divided by the ordinary curvature of $\partial K$ at the unique point with outward normal $\nu$. 
Theorem 1. Suppose $K \subset \mathbb{R}^{2}$ is a compact strictly convex set with zero in its interior, such that $\partial K$ is $C^{k, \alpha}(k \geq 2,0 \leq \alpha \leq 1)$, with positive curvature except at a finite number of points. Also suppose that $U \subset \mathbb{R}^{2}$ is a bounded open set, with piecewise $C^{k, \alpha}$ boundary which satisfies Assumption 1. and only has nonreentrant corners. Let $x \in U-R_{K, 0}$, and let $y=y(x)$ be the unique $d_{K}$-closest point to $x$ on $\partial U$. If

$$
\kappa_{K}(y(x)) d_{K}(x) \neq 1,
$$

then $d_{K}=d_{K}(\cdot, \partial U)$ is $C^{k, \alpha}$ around $x$. Furthermore, if $\nu$ is an inward normal to $\partial U$ at $y$, and $\zeta$ is a unit vector orthogonal to the segment $] x, y[$, we have

$$
\begin{aligned}
& D d_{K}(x)=\frac{\nu}{\gamma^{\circ}(\nu)}, \\
& \Delta d_{K}(x)=\frac{-\kappa(y)|\nu|^{3}\left|D \gamma^{\circ}(\nu)\right|^{2}}{\gamma^{\circ}(\nu)^{3}\left(1-\kappa_{K}(y) d_{K}(x)\right)}, \\
& D_{v w}^{2} d_{K}(x)=\Delta d_{K}(x)\langle v, \zeta\rangle\langle w, \zeta\rangle .
\end{aligned}
$$

Here, $\kappa$ is the ordinary curvature, and $\kappa_{K}$ is the $K$-curvature of $\partial U$; and $v, w$ are arbitrary vectors in $\mathbb{R}^{2}$.

Proof. The set $L:=x-d_{K}(x) K$ is inside $\bar{U}$ and touches $\partial U$ only at $y$. Since $\partial K$ is $C^{1}, y$ is not a nonreentrant corner of $\partial U$.

Let $\nu$ be an inward normal to $\partial U$. Note that $\nu(y)$ is also an inward normal to $\partial L$ at $y$. We claim that

$$
\frac{x-y}{\gamma(x-y)}=D \gamma^{\circ}(\nu(y))
$$

Note that $\xi:=\frac{x-y}{\gamma(x-y)} \in \partial K$. Hence by (2.7) we have

$$
D \gamma^{\circ}(D \gamma(\xi))=\xi
$$

But $D \gamma(\xi)$, which is nonzero, is an outward normal to $\partial K$ at $\xi$. The reason is that $\partial K=\{\gamma=1\}$, and $\gamma$ increases as we move to the outside of $K$. On the other hand, $-\nu(y)$ is an inward normal to $\partial(x-L)$ at $x-y$, and consequently an inward normal to $\partial K$ at $\xi$. Hence due to the positive 0-homogeneity of $D \gamma^{\circ}$ we get (3.3). Note that $\nu$ need not be unit for (3.3) to hold.

As a consequence of (3.3), we have

$$
x=y(x)+d_{K}(x) D \gamma^{\circ}(\nu(y)) .
$$

Note that (3.3) holds even if $x \in R_{K, 0}$ and $y$ is one of the $d_{K}$-closest points to $x$ on $\partial U$ (or even when $y$ is a reentrant corner and $\nu(y)$ is an inward normal to $\partial L$ at $y$ ). Thus, formula (3.4) holds in these cases too.

Let us show that if $\frac{\nu(y)}{|\nu(y)|} \in\left\{\mu_{1}, \ldots, \mu_{m}\right\}$, then $\kappa(y) \leq 0$. Thus by Assumption 11, $\partial U$ must be a line segment around $y$. To see this, note that $L$ is tangent to $\partial U$ at $y$, and $L-\{y\} \subset U$. This implies that the curvature of $\partial L$ at $y$, which is zero, cannot be less than the curvature of $\partial U$ at $y$.

Let $t \mapsto\left(y_{1}(t), y_{2}(t)\right)$ for $|t|<\beta$ be a smooth nondegenerate parametrization of $\partial U$ around $y$, with $\left(y_{1}(0), y_{2}(0)\right)=y$. Also suppose that the direction of the parametrization is such that $\nu(t):=\left(-y_{2}^{\prime}(t), y_{1}^{\prime}(t)\right)$ is an inward normal to $\partial U$. We can take $\beta$ small enough to ensure that 
by Assumption 1 and the above paragraph, $\nu$ is not a positive multiple of any of $\mu_{i}$ 's unless it is constant.

Consider the map

$$
F:(t, d) \mapsto\left(y_{1}(t), y_{2}(t)\right)+d D \gamma^{\circ}\left(-y_{2}^{\prime}(t), y_{1}^{\prime}(t)\right)
$$

from the open set $(-\beta, \beta) \times(0, \infty)$ into $\mathbb{R}^{2}$. We have $F\left(0, d_{K}(x)\right)=x$. We wish to compute $D F$ around this point. Note that $D \gamma^{\circ}(\nu(t))$ is differentiable with respect to $t$. Now we have

$$
D F(t, d)=\left[\begin{array}{ll}
y_{1}^{\prime}+\left[-y_{2}^{\prime \prime} D_{11}^{2} \gamma^{\circ}+y_{1}^{\prime \prime} D_{12}^{2} \gamma^{\circ}\right] d & D_{1} \gamma^{\circ} \\
y_{2}^{\prime}+\left[-y_{2}^{\prime \prime} D_{12}^{2} \gamma^{\circ}+y_{1}^{\prime \prime} D_{22}^{2} \gamma^{\circ}\right] d & D_{2} \gamma^{\circ}
\end{array}\right]
$$

Consequently

$$
\begin{aligned}
\operatorname{det} D F=-y_{2}^{\prime} D_{1} \gamma^{\circ}+y_{1}^{\prime} D_{2} \gamma^{\circ} & -d\left[-y_{2}^{\prime \prime}\left(D_{1} \gamma^{\circ} D_{12}^{2} \gamma^{\circ}-D_{2} \gamma^{\circ} D_{11}^{2} \gamma^{\circ}\right)+y_{1}^{\prime \prime}\left(D_{1} \gamma^{\circ} D_{22}^{2} \gamma^{\circ}-D_{2} \gamma^{\circ} D_{12}^{2} \gamma^{\circ}\right)\right] \\
& \quad\left\langle\nu, D \gamma^{\circ}(\nu)\right\rangle-d\left\langle D^{\perp} \gamma^{\circ}(\nu), D^{2} \gamma^{\circ}(\nu) \nu^{\prime}\right\rangle \\
= & \gamma^{\circ}(\nu)\left(1-\kappa_{K} d\right) .
\end{aligned}
$$

Here, we used (2.6), (3.1).

Now if we assume that $\kappa_{K}\left(y\left(x_{0}\right)\right) d_{K}\left(x_{0}\right) \neq 1$ for some $x_{0} \in U-R_{K, 0}$, then $F$ is $C^{k-1, \alpha}$ around $\left(0, d_{K}\left(x_{0}\right)\right)$ with a $C^{k-1, \alpha}$ inverse. Since $F:(t, d) \mapsto x$ is invertible in a neighborhood of $\left(0, d_{K}\left(x_{0}\right)\right)$, we have

$$
x=F(t(x), d(x))=y(t(x))+d(x) D \gamma^{\circ}(\nu(t(x))) .
$$

We also know that in general

$$
x=y(x)+d_{K}(x) D \gamma^{\circ}(\nu(y(x))) .
$$

If we take $x$ close enough to $x_{0}$, then by continuity $y(x), d_{K}(x)$ will be close to $y\left(x_{0}\right), d_{K}\left(x_{0}\right)$ (here we use Lemma 3 and the fact that $x \notin R_{K, 0}$ ), and by invertibility of $F$ we get

$$
y(x)=y(t(x)), \quad d_{K}(x)=d(x) .
$$

As we showed that $x \mapsto(t, d)$ is locally $C^{k-1, \alpha}$, we obtain that $d_{K}(x)$ and $y(x)$ are also locally $C^{k-1, \alpha}$.

Note that the above also shows that all points around $x_{0}$ have a unique $d_{K}$-closest point around $y\left(x_{0}\right)$, which by continuity is the unique $d_{K}$-closest point to them on $\partial U$. Thus, a neighborhood of $x_{0}$ is in $U-R_{K, 0}$. This can also be seen from the fact that $d_{K}$ is differentiable around $x_{0}$.

We can easily compute

$$
D F^{-1}=\frac{1}{\gamma^{\circ}(\nu)\left(1-\kappa_{K} d\right)}\left[\begin{array}{cc}
D_{2} \gamma^{\circ} & -D_{1} \gamma^{\circ} \\
-y_{2}^{\prime}-\left[-y_{2}^{\prime \prime} D_{12}^{2} \gamma^{\circ}+y_{1}^{\prime \prime} D_{22}^{2} \gamma^{\circ}\right] d & y_{1}^{\prime}+\left[-y_{2}^{\prime \prime} D_{11}^{2} \gamma^{\circ}+y_{1}^{\prime \prime} D_{12}^{2} \gamma^{\circ}\right] d
\end{array}\right]
$$

Using (3.1) we can simplify this as

$$
D F^{-1}=\frac{1}{\gamma^{\circ}(\nu)\left(1-\kappa_{K} d\right)}\left[\begin{array}{c}
-D^{\perp} \gamma^{\circ} \\
\nu+d\left(D^{2} \gamma^{\circ} \nu^{\prime}\right)^{\perp}
\end{array}\right]=\left[\begin{array}{c}
-\frac{D^{\perp} \gamma^{\circ}}{\gamma^{\circ}(\nu)\left(1-\kappa_{K} d\right)} \\
\frac{\nu}{\gamma^{\circ}(\nu)}
\end{array}\right] .
$$


Which implies

$$
\begin{aligned}
& D d_{K}(x)=\frac{\nu}{\gamma^{\circ}(\nu)}=\frac{\nu(t(x))}{\gamma^{\circ}(\nu(t(x)))}, \\
& D t(x)=-\frac{D^{\perp} \gamma^{\circ}(\nu)}{\gamma^{\circ}(\nu)\left(1-\kappa_{K}(y) d_{K}(x)\right)} .
\end{aligned}
$$

Consequently, since $\nu, t$ are $C^{k-1, \alpha}$ functions and $\gamma^{\circ}$ is $C^{k, \alpha}$ on the image of $\nu$ (otherwise $\frac{\nu}{\gamma^{\circ}(\nu)}$ is constant), $d_{K}$ is $C^{k, \alpha}$.

By differentiating $d_{K}$ one more time, for $i=1,2$ we get

$$
D_{i i} d_{K}=\left[\frac{\nu_{i}^{\prime}}{\gamma^{\circ}(\nu)}-\frac{\nu_{i}\left\langle D \gamma^{\circ}(\nu), \nu^{\prime}\right\rangle}{\gamma^{\circ}(\nu)^{2}}\right] D_{i} t
$$

Hence

$$
\begin{aligned}
\Delta d_{K}= & \left.\nu_{1}^{\prime} \gamma^{\circ}(\nu)-\nu_{1}\left\langle D \gamma^{\circ}(\nu), \nu^{\prime}\right\rangle\right] \frac{D_{2} \gamma^{\circ}(\nu)}{\gamma^{\circ}(\nu)^{3}\left(1-\kappa_{K} d_{K}\right)} \\
& -\left[\nu_{2}^{\prime} \gamma^{\circ}(\nu)-\nu_{2}\left\langle D \gamma^{\circ}(\nu), \nu^{\prime}\right\rangle\right] \frac{D_{1} \gamma^{\circ}(\nu)}{\gamma^{\circ}(\nu)^{3}\left(1-\kappa_{K} d_{K}\right)} \\
= & -\frac{\left\langle D^{\perp} \gamma^{\circ}, \nu^{\prime}\right\rangle \gamma^{\circ}(\nu)-\left\langle D \gamma^{\circ}, \nu^{\prime}\right\rangle\left\langle D^{\perp} \gamma^{\circ}, \nu\right\rangle}{\gamma^{\circ}(\nu)^{3}\left(1-\kappa_{K} d_{K}\right)} .
\end{aligned}
$$

Now as $\gamma^{\circ}(\nu)=\left\langle D \gamma^{\circ}, \nu\right\rangle$, the numerator of the above fraction can be written as

$$
\left\langle v^{\perp}, \nu^{\prime}\right\rangle\langle v, \nu\rangle-\left\langle v, \nu^{\prime}\right\rangle\left\langle v^{\perp}, \nu\right\rangle,
$$

where $v:=D \gamma^{\circ}(\nu)$. Since $v, v^{\perp}$ are orthogonal and have the same length, this expression is nothing but $|v|^{2}\left\langle\nu^{\prime}, \nu^{\perp}\right\rangle$. Therefore using the fact that $\left\langle\nu^{\prime}, \nu^{\perp}\right\rangle=\kappa|\nu|^{3}$ we get the desired result.

Now, let $\tilde{\xi}:=\frac{x-y(x)}{|x-y(x)|}$, and $\zeta:=-\tilde{\xi}^{\perp}$. Then as $D d_{K}$ is constant along the segment $] x, y(x)[$, we have $D_{\tilde{\xi} \tilde{\xi}}^{2} d_{K}(x)=D_{\tilde{\xi} \zeta}^{2} d_{K}(x)=0$. Also as $\tilde{\xi}, \zeta$ form an orthonormal basis, we have

$$
\Delta d_{K}(x)=D_{\tilde{\xi} \tilde{\xi}}^{2} d_{K}(x)+D_{\zeta \zeta}^{2} d_{K}(x)=D_{\zeta \zeta}^{2} d_{K}(x) .
$$

Therefore, by changing the coordinates from the orthonormal basis $\tilde{\xi}, \zeta$ to the standard basis, we get

$$
D^{2} d_{K}(x)=\left[\begin{array}{ll}
\tilde{\xi}_{1} & \zeta_{1} \\
\tilde{\xi}_{2} & \zeta_{2}
\end{array}\right]\left[\begin{array}{cc}
0 & 0 \\
0 & \Delta d_{K}(x)
\end{array}\right]\left[\begin{array}{cc}
\tilde{\xi}_{1} & \tilde{\xi}_{2} \\
\zeta_{1} & \zeta_{2}
\end{array}\right] .
$$

By applying both sides of this equation to two vectors $v=\left(v_{1}, v_{2}\right), w=\left(w_{1}, w_{2}\right)$, we obtain (3.2).

3.1. Domains with reentrant corners. Now, we allow some of the vertices of $\partial U$ to be reentrant corners. The main difference with the previous case, is that reentrant corners can be the $d_{K}$-closest point on $\partial U$ to some points in $U$. Let us first introduce a new notion.

Definition 4. The inward $\boldsymbol{K}$-normal at a point $y \in S_{i} \subset \partial U$ is

$$
\nu_{K}(y):=D \gamma^{\circ}(\nu(y))
$$

where $\nu(y)$ is an inward normal to $S_{i}$ at $y$. 
The value of $\nu_{K}$ is independent of the length of $\nu$ due to the 0 -homogeneity of $D \gamma^{\circ}$. Also, we have $\gamma\left(\nu_{K}\right)=1$ and

$$
\left\langle\nu_{K}, \nu\right\rangle=\gamma^{\circ}(\nu)>0
$$

by (2.6), (2.4). In particular, $\nu_{K}$ is really pointing inward. Note that at a corner we have two inward $K$-normals.

The motivation for this definition is that by (3.3), $\nu_{K}(y)$ is the direction along which points inside $U$ and close to $y$ have $y$ as the $d_{K}$-closest point on $\partial U$, if $y$ is the $d_{K}$-closest point to any point inside $U$.

Theorem 2. Suppose $K \subset \mathbb{R}^{2}$ is a compact strictly convex set with zero in its interior, such that $\partial K$ is $C^{k, \alpha}(k \geq 2,0 \leq \alpha \leq 1)$, with positive curvature except at a finite number of points. Also suppose that $U \subset \mathbb{R}^{2}$ is a bounded open set, with piecewise $C^{k, \alpha}$ boundary which satisfies Assumption 1. Let $x \in U-R_{K, 0}$, and let $y=y(x)$ be the unique $d_{K}$-closest point to $x$ on $\partial U$. If $y$ is not a reentrant corner and

$$
\kappa_{K}(y(x)) d_{K}(x) \neq 1,
$$

then $d_{K}=d_{K}(\cdot, \partial U)$ is $C^{k, \alpha}$ around $x$. Furthermore, $D d_{K}, D^{2} d_{K}$ at $x$ are given by (3.2).

If $y$ is a strict reentrant corner and $x-y$ is not parallel to one of the inward $K$-normals at $y$, then

$$
d_{K}(z)=\gamma(z-y)
$$

for $z$ close to $x$. Thus $d_{K}$ is $C^{k, \alpha}$ around $x$. And, if $x-y$ is parallel to one of the inward $K$-normals at $y$ and $\kappa_{K}(y) d_{K}(x) \neq 1$, where $\kappa_{K}$ is the $K$-curvature of the corresponding boundary part, then $d_{K}$ is $C^{1,1}$ around $x$ (but not $C^{2}$ in general).

Finally, if $y$ is a non-strict reentrant corner and $d_{K}(x) \neq \frac{1}{\kappa_{K, 1}}, \frac{1}{\kappa_{K, 2}}$, where $\kappa_{K, 1}, \kappa_{K, 2}$ are the $K$-curvatures at $y$ from different sides, then $d_{K}$ is $C^{1,1}$ around $x$ (but not $C^{2}$ in general).

Proof. If $y$ is not a reentrant corner, the proof is the same as in Theorem 1; so we assume that $y \in S_{1} \cap S_{2}$ is a reentrant corner. Consider the set $L:=x-d_{K}(x) K$ which is inside $\bar{U}$ and touches $\partial U$ only at $y$. Note that $y \in \partial L$. Let $\nu$ be the inward unit normal to $\partial L$ at $y$.

First suppose that $y$ is a strict reentrant corner. Let $\nu_{1}, \nu_{2}$ be the inward unit normals to $S_{1}, S_{2}$ at $y$. Then, $\nu$ must lie between $\nu_{1}, \nu_{2}$ or coincide with one of them, otherwise $L$ would intersect the exterior of $U$. If $x-y$ is not parallel to one of the inward $K$-normals at $y$, then $\nu \neq \nu_{1}, \nu_{2}$ by (3.3). We need to show that

$$
d_{K}(z)=\gamma(z-y)
$$

for $z$ close to $x$.

To prove this, it is enough to show that $L_{z}:=z-\gamma(z-y) K$ is a subset of $\bar{U}$ for $z$ close to $x$. Suppose to the contrary that there exists a sequence $z_{i} \rightarrow x$ such that $L_{z_{i}}$ intersects $\mathbb{R}^{2}-\bar{U}$ at $y_{i}$. Due to the compactness of $K$ we can assume that $y_{i}$ converges to some limit. But that limit must belong to $L$, and it cannot be an interior point of $U$; hence we must have $y_{i} \rightarrow y$. On the other hand, $L_{z_{i}}$ lies on one side of the tangent line to $L_{z_{i}}$ at $y$, and that line is close to $l_{y}$, the tangent line to $L$ at $y$. Now, consider two half-lines with vertex $y$ which are between $l_{y}$ and $S_{1}, S_{2}$ respectively. Then for large enough $i, L_{z_{i}}$ and $L$ are on the same side of the union of these two half-lines. But this contradicts the fact that $y_{i}$ is in the intersection of a neighborhood of $y$ and $\mathbb{R}^{2}-\bar{U}$. 
Next consider the case where $\nu=\nu_{1}$. Then $x-y$ is parallel to the $K$-normal to $S_{1}$ at $y$. Note that if $\nu_{1}$ coincides with one of the $\mu_{i}$ 's, then $S_{1}$ must be a line segment by Assumption 1 otherwise $L$ cannot be tangent to $S_{1}$ at $y$ and lies inside $\bar{U}$. Consider a small ball around $x$ divided by $l$, the line passing through $x, y$. Denote by $B$ the open side of the ball which is in the same side of $l$ as $S_{1}$. First note that by Lemma 3 , the $d_{K}$-closest points on $\partial U$ to points in $B$ must be close to $y$; so they either lie on $S_{1}$ or $S_{2}$. But if $B$ is small enough, those $d_{K}$-closest points cannot belong to $S_{2}$.

To see this, suppose to the contrary that $w_{i} \in S_{2}$ is $d_{K}$-closest to $z_{i} \in B$, and $z_{i} \rightarrow x$. First let us assume that $w_{i} \in S_{2}-\{y\}$. Then, by Lemma 3 we know that $w_{i} \rightarrow y$. Also by (3.3) we have

$$
\frac{z_{i}-w_{i}}{\gamma\left(z_{i}-w_{i}\right)}=D \gamma^{\circ}\left(\nu\left(w_{i}\right)\right)
$$

The left hand side of this equality converges to $\frac{x-y}{\gamma(x-y)}$ which equals $D \gamma^{\circ}\left(\nu_{1}\right)$, while the right hand side converges to $D \gamma^{\circ}\left(\nu_{2}\right)$. Now, Corollary 1.7.3 of [13] says that for some unit vector $\tilde{\nu}, D \gamma^{\circ}(\tilde{\nu})$ is the unique point on $\partial K$ which has $\tilde{\nu}$ as the outward unit normal. Since $\partial K$ is $C^{1}$, this implies that $D \gamma^{\circ}$ is injective on the unit circle; thus we arrive at a contradiction.

Now let us show that $w_{i}$ cannot equal $y$ for any $i$. If this happens, the definition of $B$ and (3.3) imply that the inward unit normal to $L_{z_{i}}$ at $y, \nu\left(w_{i}\right)$, lies between $\nu_{1}$ and $-\nu_{1}^{\perp}$. The reason is that $D \gamma^{\circ}$ is orientation preserving on the unit circle due to the convexity of $\gamma^{\circ}$. In other words

$$
\left\langle D \gamma^{\circ}\left(\nu\left(w_{i}\right)\right)-D \gamma^{\circ}\left(\nu_{1}\right), \nu\left(w_{i}\right)-\nu_{1}\right\rangle \geq 0 .
$$

Now, if $\nu\left(w_{i}\right)=\nu_{1}$, then $x, y, z_{i}$ must be collinear by (3.4), which is impossible by the definition of $B$; and if $\nu\left(w_{i}\right) \neq \nu_{1}$, then $L_{z_{i}}$ would intersect the exterior of $U$.

Thus far, we have shown that $B$ can be taken to be small enough so that the $d_{K}$-closest points on $\partial U$ to points in $B$ are on $S_{1}-\{y\}$. Let us also show that if $B$ is small enough, then $R_{K, 0}$ does not intersect it. Suppose to the contrary that there is a sequence $z_{i} \rightarrow x$ of elements of $B$ such that they all have more than one $d_{K}$-closest points on $S_{1}-\{y\}$. Let $w_{i, 1}, w_{i, 2}$ be two distinct $d_{K}$-closest points to $z_{i}$. First note that for this to happen, $S_{1}$ cannot be a line segment; since $K$ is strictly convex. Hence we can assume that $\nu_{1}$ is not one of the $\mu_{j}$ 's. Now, by (3.4) we have

$$
z_{i}-w_{i, 1}=d_{K}\left(z_{i}\right) D \gamma^{\circ}\left(\nu\left(w_{i, 1}\right)\right), \quad z_{i}-w_{i, 2}=d_{K}\left(z_{i}\right) D \gamma^{\circ}\left(\nu\left(w_{i, 2}\right)\right) .
$$

If we subtract these two equations we get

$$
w_{i, 1}-w_{i, 2}=-d_{K}\left(z_{i}\right)\left[D \gamma^{\circ}\left(\nu\left(w_{i, 1}\right)\right)-D \gamma^{\circ}\left(\nu\left(w_{i, 2}\right)\right)\right] .
$$

Let $t \mapsto y(t)$ be a smooth nondegenerate parametrization of $S_{1}$ around $y$ with $y(0)=y$. Then there are $t_{i, j}$ such that $w_{i, j}=y\left(t_{i, j}\right)$. Since $w_{i, 1}, w_{i, 2} \rightarrow y$, we have $t_{i, 1}, t_{i, 2} \rightarrow 0^{+}$. As $D \gamma^{\circ}$ is differentiable at $\nu_{1}$, we can divide by $t_{i, 1}-t_{i, 2}$ and let $i \rightarrow \infty$ in (3.6) to get

$$
y^{\prime}(0)=-d_{K}(x)\left[D^{2} \gamma^{\circ}\left(\nu_{1}\right) \nu^{\prime}(0)\right] \text {. }
$$

By using (3.1) and the fact that $y^{\prime}(0)=-\nu_{1}^{\perp}$, we get

$$
\left(1-\kappa_{K}(y) d_{K}(x)\right) \nu_{1}^{\perp}=0 .
$$

Which is a contradiction.

We assumed that $1-\kappa_{K}(y) d_{K}(x) \neq 0$, where $\kappa_{K}(y)$ is the $K$-curvature of $S_{1}$ at $y$. Let us also assume that $B$ is small enough so that for $z \in B$ we have $1-\kappa_{K}(y(z)) d_{K}(z) \neq 0$. Then, since 
$R_{K, 0} \cap B=\emptyset$, we can repeat the proof of Theorem 1 to deduce that $d_{K}$ is at least $C^{2}$ on $B$. We also have $D d_{K}(z)=\frac{\nu(y(z))}{\gamma^{\circ}(\nu(y(z)))}$ for $z \in B$.

Next, let us show that if $B$ is small enough, the points on the segment $l \cap \partial B$ have $y$ as the only $d_{K}$-closest point on $\partial U$. This is obvious for points in $] x, y[$ by Lemma 1, so we only need to consider points $z$ on $l \cap \partial B$ such that $x \in] z, y\left[\right.$. Take a sequence $z_{i} \in B$ that converges to $z$. Then we can find points $\left.x_{i} \in\right] z_{i}, y\left(z_{i}\right)$ [ such that $x_{i} \rightarrow x$. Since we have $y\left(z_{i}\right)=y\left(x_{i}\right) \rightarrow y, y$ is one of the $d_{K}$-closest points on $\partial U$ to $z$ by Lemma 3 . Thus $y$ is the only $d_{K}$-closest point on $\partial U$ to points in $] z, y[$; and we can make $B$ small enough to have the aforementioned property. We also make $B$ small enough so that $1-\kappa_{K} d_{K} \neq 0$ on $\bar{B}$.

Now we claim that $D d_{K}$ is uniformly continuos on $B$. Thus it admits continuous extension to $\bar{B}$. It is enough to show that $D d_{K}(z)$ has a limit as $z$ approaches $\partial B$. Since we can make $B$ smaller, we only need to consider $l \cap \partial B$. Suppose $z_{i} \in B$ converge to $z$ on $l \cap \partial B$. Then $y\left(z_{i}\right) \rightarrow y$ and

$$
D d_{K}\left(z_{i}\right) \rightarrow \frac{\nu_{1}}{\gamma^{\circ}\left(\nu_{1}\right)}
$$

Also note that $d_{K}$ is a linear function on $l \cap \partial B$, and its derivative along $l$ is precisely the projection of $\frac{\nu_{1}}{\gamma^{\circ}\left(\nu_{1}\right)}$ onto $l$. Therefore, $d_{K}$ is $C^{1}$ on $\bar{B}$.

Let $z \in l \cap \partial B$. Then $D d_{K}(z)=\frac{\nu_{1}}{\gamma^{\circ}\left(\nu_{1}\right)}$ from the side of $B$. Let us compute $D d_{K}(z)$ from the other side of $l$. We know that on the other side of $l, d_{K}(\cdot)=\gamma(\cdot-y)$. Hence $D d_{K}(z)=D \gamma(z-y)$. Now we have $z-y=d_{K}(z) D \gamma^{\circ}\left(\nu_{1}\right)$ by Lemma (3.4); so by (2.5), (2.7) we get

$$
D d_{K}(z)=D \gamma\left(d_{K}(z) D \gamma^{\circ}\left(\nu_{1}\right)\right)=D \gamma\left(D \gamma^{\circ}\left(\nu_{1}\right)\right)=\frac{\nu_{1}}{\gamma^{\circ}\left(\nu_{1}\right)} \text {. }
$$

Therefore $D d_{K}$ is continuous on $l \cap \partial B$ from both sides, and thence $d_{K}$ is $C^{1}$ around $x$.

As $d_{K}$ is $C^{2}$ on both sides of $l \cap \partial B$, to show that it is $C^{1,1}$ around $x$, it is enough to show that $D^{2} d_{K}$ remains bounded as we approach $l \cap \partial B$ from either side. This is obvious on the side of $l$ where $d_{K}(\cdot)=\gamma(\cdot-y)$. Let us consider the side where $B$ lies. It suffices to show that

$$
\operatorname{tr}\left[\left(D^{2} d_{K}\right)^{2}\right]=\left(D_{11}^{2} d_{K}\right)^{2}+\left(D_{22}^{2} d_{K}\right)^{2}+2\left(D_{12}^{2} d_{K}\right)^{2}
$$

has limit as we approach $l \cap \partial B$. As shown in the proof of Theorem 1, the matrix of $D^{2} d_{K}$ in the standard basis is similar to the matrix

$$
\left[\begin{array}{cc}
0 & 0 \\
0 & \Delta d_{K}
\end{array}\right]
$$

Since, the trace of similar matrices are the same, we get

$$
\operatorname{tr}\left[\left(D^{2} d_{K}\right)^{2}\right]=\left(\Delta d_{K}\right)^{2} .
$$

Now if $z_{i} \in B$ approach $l \cap \partial B$, then $y\left(z_{i}\right) \rightarrow y$ and $\nu\left(y\left(z_{i}\right)\right) \rightarrow \nu_{1}$. Thus, as $1-\kappa_{K} d_{K} \neq 0$ on $\bar{B}$, $\Delta d_{K}\left(z_{i}\right)$ has a limit by (3.2).

To see that $d_{K}$ is not $C^{2}$ around $x$ in general, we can compute $\Delta d_{K}$ from both sides of $l$, and see that in simple examples they do not agree on $l$. For example, when $K$ is the unit disk around the origin and $S_{1}$ is a line segment, we see this phenomenon.

When $y$ is a non-strict reentrant corner, the argument is similar to the above. 
Theorem 3. Suppose $K, U$ satisfy the same assumptions as in Theorem 国 Let $y \in S_{i} \subset \partial U$, and suppose that it is not a corner. Also suppose that $S_{i}$ is a line segment if $\nu(y)=c \mu_{j}$ for some $c>0$. Then for some $r>0$ we have

$$
B_{r}(y) \cap R_{K}=\emptyset .
$$

Furthermore, $d_{K}$ is at least $C^{2}$ up to $\partial U \cap B_{r}(y)$.

Proof. First we claim that for some $r>0$ we have

$$
B_{r}(y) \cap R_{K, 0}=\emptyset .
$$

This is easy to show when $S_{i}$ is a line segment. Hence we assume that $\nu(y)$, and consequently $\nu$ around $y$, is not a positive multiple of any of $\mu_{j}$ 's. Since $\partial U$ is at least $C^{2}$ around $y$, we can inscribe circles in $U$ which are tangent to $\partial U$ and touch it only at one point near $y$. We can also assume that the radii of these circles have a positive lower bound. Now we can inscribe sets of the form $x-r K$ in each of these circles so that it touches $\partial U$ at the same point that the circle does. We can also assume that these $r$ 's have a positive lower bound. The reason is that $\partial K$ has positive curvature except at a finite number of points, and those points are excluded by our assumption. The existence of such inscribed sets implies the claim easily. Note that as a consequence, $y$ is the $d_{K}$-closest point on $\partial U$ to some points in $U$.

Another way to prove this claim, is to assume the existence of a sequence $x_{i} \in R_{K, 0}$ that converges to $y$, and arrive at a contradiction as we did in the proof of Theorem 2 .

Now note that $\kappa_{K}$ is continuous, and hence bounded, on $\partial U$ around $y$. Thus, as $y(x) \rightarrow y$ when $x \rightarrow y$ by Lemma 3, we can make $r$ small enough so that $\kappa_{K}(y(x)) d_{K}(x) \neq 1$ for $x \in U \cap B_{r}(y)$. Therefore we have $B_{r}(y) \cap R_{K}=\emptyset$.

Next we show that $d_{K}$ is at least $C^{2}$ up to $\partial U \cap B_{r}(y)$. To prove this, it is enough to show that $D d_{K}, D^{2} d_{K}$ have limits as we approach $y$. Take $x \in U \cap B_{r}(y)$. Then $D d_{K}(x)=\frac{\nu(y(x))}{\gamma^{\circ}(\nu(y(x)))}$. When $x \rightarrow y$ we have $y(x) \rightarrow y$, so by continuity of $\nu$ we get $D d_{K}(x) \rightarrow \frac{\nu(y)}{\gamma^{\circ}(\nu(y))}$ as desired.

To show the same for $D^{2} d_{K}$, we use (3.2) and the continuity of $\kappa, \kappa_{K}$ on $\partial U \cap B_{r}(y)$, to get

$$
\Delta d_{K}(x) \rightarrow \frac{-\kappa(y)|\nu(y)|^{3}\left|D \gamma^{\circ}(\nu(y))\right|^{2}}{\gamma^{\circ}(\nu(y))^{3}}
$$

On the other hand we have

$$
\zeta(x):=\left(\frac{x-y(x)}{|x-y(x)|}\right)^{\perp}=\left(\frac{D \gamma^{\circ}(\nu(y(x)))}{\left|D \gamma^{\circ}(\nu(y(x)))\right|}\right)^{\perp} \rightarrow\left(\frac{D \gamma^{\circ}(\nu(y))}{\left|D \gamma^{\circ}(\nu(y))\right|}\right)^{\perp} .
$$

Note that here we used (3.3). Hence again by (3.2) we see that $D^{2} d_{K}(x)$ has a limit as $x \rightarrow y$.

Remark 5. When $\nu(y)$ is a positive multiple of a $\mu_{j}$ and the curvature of $S_{i}$ is positive at $y, R_{K, 0}$ can have $y$ as a limit point. The same thing happens when $y$ is a nonreentrant corner. When $y$ is a strict reentrant corner and we approach it from the region between its inward $K$-normals, $D d_{K}$ will not have a limit and $D^{2} d_{K}$ will blow up, by (2.5).

Finally, when $y$ is a non-strict reentrant corner, with a slight modification of the above proof we can show that $R_{K}$ has a positive distance from $y$, and $d_{K}$ is $C^{1}$ up to $y$. 


\section{Characterizing the Ridge}

At this point we have the tools to specify the points in the $d_{K}$-ridge of $U$.

Theorem 4. Suppose $K, U$ satisfy the same assumptions as in Theorem 2 . Then the $d_{K}$-ridge consists of $R_{K, 0}$ and those points $x$ outside of it at which

$$
\kappa_{K}(y(x)) d_{K}(x)=1 .
$$

Here, if $y=y(x)$ is a reentrant corner, then $x-y$ must be parallel to one of the inward $K$-normals at $y$, and $\kappa_{K}$ is the $K$-curvature of the corresponding boundary part.

Proof. So far, we showed that $R_{K}$ contains $R_{K, 0}$. We also showed in Theorems 1 , 2 that every point outside $R_{K, 0}$ which is not described in the statement of the theorem is not in $R_{K}$, i.e. those points at which $1-\kappa_{K} d_{K} \neq 0$, and those points between the $K$-normals of a strict reentrant corner which have that corner as the $d_{K}$-closest point.

Now to prove theorem's assertion, first suppose that $y \in S_{1} \cap S_{2}$ is a reentrant corner and $1-\kappa_{K}(y) d_{K}(x)=0$, where $\kappa_{K}$ is the $K$-curvature of $S_{1}$. Then $\kappa_{K}(y)=\frac{1}{d_{K}(x)}>0$, and consequently $\kappa(y)>0$, where $\kappa$ is the ordinary curvature of $S_{1}$. Consider the line segment ] $x, y$ [. On this segment, $y$ is the unique $d_{K}$-closest point on $\partial U$; so $d_{K}$ decreases linearly as we move from $x$ to $y$. Hence $1-\kappa_{K} d_{K}>0$ on ] $x, y$ [. Thus, as seen in the proof of Theorem 2, $d_{K}$ is at least $C^{2}$ on an open set $B$, which is on one side of $] x, y$ [ and has $] x, y$ [ as part of its boundary. Also, the $d_{K}$-closest points to points of $B$ lie on $S_{1}$. Choose a sequence $z_{i} \in B$ that converges to $x$. Then $y\left(z_{i}\right) \rightarrow y$ by Lemma 3. and by continuity of $\kappa_{K}, \kappa$ on $S_{1}$ we have

$$
\kappa_{K}\left(y\left(z_{i}\right)\right) \rightarrow \kappa_{K}(y), \quad \kappa\left(y\left(z_{i}\right)\right) \rightarrow \kappa(y) .
$$

Thus in particular, $\kappa\left(y\left(z_{i}\right)\right)>0$ for $i$ large enough. Since on $B, \Delta d_{K}$ is given by (3.2), $\Delta d_{K}\left(z_{i}\right)$ blows up as $z_{i} \rightarrow x$. Therefore, $d_{K}$ can not be $C^{1,1}$ in any neighborhood of $x$.

If $y$ is not a reentrant corner, we can repeat the above argument by simply approaching $x$ through points of $] x, y[$.

The proof of the following theorem is a variant of the proof of a similar result in [4].

Theorem 5. Suppose $K, U$ satisfy the same assumptions as in Theorem Q Then for $x \in U-R_{K}$ we have

$$
1-\kappa_{K}(y(x)) d_{K}(x)>0 .
$$

Here, if $y=y(x)$ is a reentrant corner, then $x-y$ must be parallel to one of the inward $K$-normals at $y$, and $\kappa_{K}$ is the $K$-curvature of the corresponding boundary part.

Proof. We will show that $1-\kappa_{K}(y) d_{K}(x) \geq 0$. This gives the desired result, since we know that $1-\kappa_{K}(y) d_{K}(x) \neq 0$. If $\kappa_{K}(y)=0$ the relation holds trivially, so suppose it is nonzero. Note that as shown in the proof of Theorem 1, $y$ cannot be a nonreentrant corner, and the inward unit normal to $\partial U$ at $y$ is not equal to any of the $\mu_{i}$ 's, since we assumed that $\kappa_{K}(y) \neq 0$.

Let $t \mapsto y(t)$ be a smooth nondegenerate parametrization of a segment of $\partial U$ around $y$ which has $y$ as an endpoint, and $y(0)=y$. We assume that the direction of the parametrization is such that 
$\nu:=\left(y^{\prime}\right)^{\perp}$ is an inward normal to $\partial U$. Consider the function $t \mapsto \gamma(x-y(t))$. It has a minimum at $t=0$; and there, its first derivative is

$$
\left\langle D \gamma(x-y),-y^{\prime}(0)\right\rangle=\left\langle D \gamma(x-y), \nu^{\perp}\right\rangle .
$$

But by (3.4) we have $x-y=d_{K}(x) D \gamma^{\circ}(\nu)$. Hence by (2.5), (2.7), the first derivative vanishes at $t=0$. Thus the second derivative must be nonnegative at $t=0$, i.e.

$$
\left\langle D^{2} \gamma(x-y) y^{\prime}(0), y^{\prime}(0)\right\rangle-\left\langle D \gamma(x-y), y^{\prime \prime}(0)\right\rangle \geq 0 .
$$

By using homogeneity of $D \gamma, D^{2} \gamma$, and (2.7), (3.4) we get

$$
\frac{1}{d_{K}(x)}\left\langle D^{2} \gamma\left(D \gamma^{\circ}(\nu)\right) \nu^{\perp}, \nu^{\perp}\right\rangle+\left\langle\frac{\nu}{\gamma^{\circ}(\nu)},\left(\nu^{\prime}\right)^{\perp}\right\rangle \geq 0 \text {. }
$$

On the other hand, by differentiating (2.7) we get

$$
\sum_{k} D_{i k}^{2} \gamma\left(D \gamma^{\circ}(\nu)\right) D_{k j}^{2} \gamma^{\circ}(\nu)=\frac{1}{\gamma^{\circ}(\nu)} \delta_{i j}-\frac{\nu_{i} D_{j} \gamma^{\circ}(\nu)}{\gamma^{\circ}(\nu)^{2}} .
$$

Multiplying both sides by $\nu_{i}^{\perp}, \nu_{j}^{\prime}$ and summing over $i, j$ gives us

$$
\begin{aligned}
\left\langle D^{2} \gamma\left(D \gamma^{\circ}(\nu)\right) \nu^{\perp}, D^{2} \gamma^{\circ}(\nu) \nu^{\prime}\right\rangle & =\sum_{i, j, k} \nu_{i}^{\perp} D_{i k}^{2} \gamma\left(D \gamma^{\circ}(\nu)\right) D_{k j}^{2} \gamma^{\circ}(\nu) \nu_{j}^{\prime} \\
& =\frac{1}{\gamma^{\circ}(\nu)}\left\langle\nu^{\perp}, \nu^{\prime}\right\rangle .
\end{aligned}
$$

And by (3.1) we obtain

$$
\left\langle D^{2} \gamma\left(D \gamma^{\circ}(\nu)\right) \nu^{\perp}, \nu^{\perp}\right\rangle=\frac{1}{\kappa_{K}(y) \gamma^{\circ}(\nu)}\left\langle\nu^{\perp}, \nu^{\prime}\right\rangle
$$

If we insert this in (4.1) and use the fact that the ordinary curvature is given by $\kappa=\frac{\left\langle\nu^{\perp}, \nu^{\prime}\right\rangle}{|\nu|^{3}}$, we deduce that

$$
0 \leq \frac{1}{\kappa_{K}(y) d_{K}(x) \gamma^{\circ}(\nu)}\left\langle\nu^{\perp}, \nu^{\prime}\right\rangle+\frac{1}{\gamma^{\circ}(\nu)}\left\langle\nu,\left(\nu^{\prime}\right)^{\perp}\right\rangle=\frac{|\nu|^{3} \kappa(y)\left[1-\kappa_{K}(y) d_{K}(x)\right]}{\kappa_{K}(y) d_{K}(x) \gamma^{\circ}(\nu)} .
$$

Now, as $\kappa, \kappa_{K}$ have the same sign, we must have $1-\kappa_{K} d_{K} \geq 0$ as desired.

Remark 6. Suppose $y$ is a non-strict reentrant corner, and $\kappa_{K, 1}>\kappa_{K, 2}$, where $\kappa_{K, 1}, \kappa_{K, 2}$ are the $K$-curvatures at $y$ from different sides. Then by Theorem 4 , if

$$
x_{i}:=y+\frac{1}{\kappa_{K, i}} \nu_{K}(y)
$$

have $y$ as the only $d_{K}$-closest point on $\partial U$, then they belong to the $d_{K}$-ridge. But, Theorem 5 implies that $y$ cannot be a $d_{K}$-closest point to any point on the segment $\left.] x_{1}, x_{2}\right]$. Thus, $x_{1}$ is the only point along the $K$-normal at $y$, that can belong to $R_{K}-R_{K, 0}$ and have $y$ as the unique $d_{K}$-closest point on $\partial U$. 


\section{REFERENCES}

[1] L. A. Caffarelli and A. Friedman. The free boundary for elastic-plastic torsion problems. Trans. Amer. Math. Soc., 252:65-97, 1979.

[2] P. T. Chruściel, J. H.G. Fu, G. J. Galloway, and R. Howard. On fine differentiability properties of horizons and applications to Riemannian geometry. J. Geom. Phys., 41(1):1 - 12, 2002.

[3] F. H. Clarke, R. J. Stern, and P. R. Wolenski. Proximal smoothness and the lower- $C^{2}$ property. J. Convex Anal., 2(1-2):117-144, 1995.

[4] G. Crasta and A. Malusa. The distance function from the boundary in a Minkowski space. Trans. Amer. Math. Soc., 359(12):5725-5759 (electronic), 2007.

[5] J. Itoh and M. Tanaka. The Lipschitz continuity of the distance function to the cut locus. Trans. Amer. Math. Soc., 353(1):21-40, 2001.

[6] Y. Li and L. Nirenberg. The distance function to the boundary, Finsler geometry, and the singular set of viscosity solutions of some Hamilton-Jacobi equations. Comm. Pure Appl. Math., 58(1):85-146, 2005.

[7] C. Mantegazza and A. C. Mennucci. Hamilton-Jacobi equations and distance functions on Riemannian manifolds. Appl. Math. Optim., 47(1):1-25, 2003.

[8] R. Poliquin, R. Rockafellar, and L. Thibault. Local differentiability of distance functions. Trans. Amer. Math. Soc., 352(11):5231-5249, 2000.

[9] M. Safdari. The free boundary of variational inequalities with gradient constraints. Nonlinear Anal. Theory Methods Appl., 123-124:1 - 22, 2015.

[10] M. Safdari. On the shape of the free boundary of variational inequalities with gradient constraints. Interfaces Free Bound., 19(2):183-200, 2017.

[11] M. Safdari. The regularity of some vector-valued variational inequalities with gradient constraints. Comm. Pure Appl. Anal., 17(2):413-428, 2018.

[12] M. Safdari. Global optimal regularity for variational problems with nonsmooth non-strictly convex gradient constraints. preprint, arxiv.org/abs/1807.01590v1, 2018.

[13] R. Schneider. Convex bodies: the Brunn-Minkowski theory, volume 151 of Encyclopedia of Mathematics and its Applications. Cambridge University Press, Cambridge, expanded edition, 2014.

[14] T. W. Ting. The ridge of a Jordan domain and completely plastic torsion. J. Math. Mech., 15: 15-47, 1966. 